\title{
When should cardiovascular prevention begin? The importance of antenatal, perinatal and primordial prevention
}

European Journal of Preventive

\author{
Flavio D'Ascenzi ',2, Carlotta Sciaccaluga', \\ Matteo Cameli', Annagrazia Cecere ${ }^{3}$, Marco M Ciccone ${ }^{3}$, \\ Simona Di Francesco ${ }^{4,5}$, Antonello Ganau, Egidio Imbalzano6, \\ Riccardo Liga ${ }^{7}$, Pietro Palermo ${ }^{8}$, Pasquale Palmiero', \\ Gianfranco Parati ${ }^{10,1}$, Roberto Pedrinelli ${ }^{7}$, Piero Scicchitano ${ }^{3}$, \\ Annapaola Zito ${ }^{6}$ and Anna V Mattioli ${ }^{12}$
}

\begin{abstract}
Cardiovascular diseases represent a major health problem, being one of the leading causes of morbidity and mortality worldwide. Therefore, in this scenario, cardiovascular prevention plays an essential role although it is difficult to establish when promoting and implementing preventive strategies. However, there is growing evidence that prevention should start even before birth, during pregnancy, aiming to avoid the onset of cardiovascular risk factors, since events that occur early in life have a great impact on the cardiovascular risk profile of an adult. The two pillars of this early preventive strategy are nutrition and physical exercise, together with prevention of cardio-metabolic diseases during pregnancy. This review attempts to gather the growing evidence of the benefits of antenatal, perinatal and primordial prevention, discussing also the possibility to reverse or to mitigate the cardiovascular profile developed in the initial stages of life. This could pave the way for future research, investigating the optimal time and duration of these preventing measures, their duration and maintenance in adulthood, and the most effective interventions according to the different age and guiding in the next years, the best clinical practice and the political strategies to cope with cardiovascular disease.
\end{abstract}

\section{Keywords}

Risk factors, children, obesity, heart disease, diabetes mellitus, exercise

Received 4 June 2019; accepted 19 November 2019

\section{Background}

Atherosclerotic cardiovascular diseases (ASCVDs) have a great impact on general health and on socioeconomic burden, being one of the leading causes of

\footnotetext{
'Department of Medical Biotechnologies, Division of Cardiology, University of Siena, Italy

${ }^{2}$ Department of Medicine, University of Pittsburgh, USA

${ }^{3}$ Cardiovascular Diseases Section, Department of Emergency and Organ Transplantation (DETO), University of Bari, Italy

${ }^{4}$ Department of Medical and Oral Sciences and Biotechnologies, G. D'Annunzio University of Chieti-Pescara, Italy

${ }^{5}$ Department of Urological, Biomedical and Translational Sciences, Federiciana University, Italy

${ }^{6}$ Department of Clinical and Experimental Medicine, University of Messina, Italy
}

morbidity and mortality worldwide. ${ }^{1}$ Therefore, the major ASCVD risk factors have relevant consequences on overall health. Over recent years, an increasing

\footnotetext{
${ }^{7}$ Cardio-Thoracic and Vascular Department, University Hospital of Pisa, Italy

${ }^{8}$ Centro Cardiologico Monzino, IRCCS, Italy

${ }^{9} \mathrm{ASL}$ Brindisi, Cardiology Equipe, District of Brindisi, Italy

${ }^{10}$ Department of Cardiovascular, Neural, and Metabolic Sciences, S. Luca

Hospital, IRCCS, Istituto Auxologico Italiano, Italy

"School of Medicine and Surgery, University Milano-Bicocca, Italy

${ }^{12}$ Department of Surgical, Medical and Dental Department of

Morphological Sciences Related to Transplant, Oncology and

Regenerative Medicine, University of Modena and Reggio Emilia, Italy

Corresponding author:

Flavio D'Ascenzi, Department of Medical Biotechnologies, Division of Cardiology, University of Siena, Viale M. Bracci, 16, 53100 Siena, Italy. Email: flavio.dascenzi@unisi.it
} 
number of chronic diseases has been observed, especially metabolic disorders such as diabetes mellitus and obesity, which are known to have a relevant impact on ASCVD risk. ${ }^{2}$ Contrary to common belief, this phenomenon does not involve only adults and the elderly, but it has been gaining relevant weight among adolescents and young adults, being able to influence each stage of life. ${ }^{3}$ Therefore, the events occurring in the early phases of life might have a great impact on the future ASCVD risk. For these reasons, ASCVD prevention should start as soon as possible, in order to avoid the onset of predisposing factors that might affect individual health later in life. Therefore, the prevention strategies that the family and the mother might follow before pregnancy and the measures that could be taken to provide the best maternal environment for the foetus (antenatal and perinatal prevention) have assumed a relevant role to avoid the onset of predisposing ASCVD risk factors together with primordial prevention, that is, the implementation of interventions set to avoid the onset of ASCVD risk factors once the baby is born. ${ }^{4}$ This review attempts to gather the growing evidence of the benefits of these prevention strategies, also discussing whether the ASCVD profile developed in the initial stages of life can be reversed or mitigated. We have based our paper on the research on PubMed literature, without posing a time limit. The primary search used the following keywords: primordial prevention, pre-eclampsia and cardiovascular risk, pre-terms and cardiovascular risk, gestational diabetes, nutrition in children, physical activity in children, childhood obesity, adolescents and cardiovascular risk. Articles not in English and not on humans were not included. Notably, this is a literature review and not a systematic review: take-home messages of the single studies have been reported but data were not extracted and analysed.

\section{The role of intra-uterine life}

There is growing evidence that events that occur during intrauterine life have a great impact on the ASCVD risk profile of the child, which might even be sustained later in life, as Barker et al. started investigating almost 30 years ago. ${ }^{5,6}$ Pregnancy-related hypertensive disorders, gestational diabetes, pre-term birth and parents' behaviour as well as physiological imbalances have emerged as the most relevant factors having an impact on the ASCVD risk profile. Indeed, there is growing evidence that parental smoking during pregnancy has an important impact on the development of cardiovascular risk factors in children ${ }^{7,8}$ and adolescents, which might be sustained even in adulthood. ${ }^{9}$ In particular, several studies have suggested the association between maternal smoking and offspring obesity, ${ }^{10}$ and in general with paediatric cardiovascular morbidity. ${ }^{11}$ Furthermore, maternal anxiety and depression during pregnancy, mainly in the last trimester, might have an impact on the health of the offspring, ${ }^{12}$ especially on the cardiovascular responses to stress. ${ }^{13}$ This highlights the importance of early ASCVD prevention, both in the mothers and in the offspring, especially in light of the possible association of pre-pregnancy factors with negative effects on the newborn, such as the connection that has been recently drawn between maternal body mass index before pregnancy and blood pressure in the offspring. ${ }^{14}$

\section{Pregnancy-related hypertensive disorders}

During pregnancy the cardiovascular system undergoes significant changes that lead to a progressive reduction of blood pressure (BP) until the trend is reverse toward the end of the gestational period, when the BP reaches the highest values. ${ }^{15}$ This physiological phenomenon guarantees an optimal placental functioning, laying the basis for a better outcome. ${ }^{16}$ On the other hand, both the mother and the offspring's outcome are negatively affected by an abnormal elevated BP during the third trimester, since that could predispose to pre-term delivery, low birth weight and pre-eclampsia, ${ }^{17,18}$ and it has been recently found associated with a higher offspring BP. ${ }^{19}$ Gestational hypertension is defined as new onset of elevated BP after the 20th week of pregnancy, whereas pre-eclampsia is a complex syndrome with elevated $\mathrm{BP}$ associated with proteinuria. Its pathophysiological basis is characterized by increased levels of antiangiogenetic factors that impair the normal placental function, which then leads to foetal hypoxia. ${ }^{20}$ It has been widely proven that this syndrome is responsible for an augmented morbidity and mortality in both the mother and the offspring ${ }^{20}$ and is associated with endothelial dysfunction ${ }^{21}$ and with changes in retinal microcirculation. ${ }^{22}$ This finding stresses the role of retinal microcirculation as an accessible site to analyse systemic human microcirculation. Indeed, changes in retinal microcirculation, such as a reduced diameter of retinal arterioles, are correlated with both systemic hypertension and ASCVD outcome, including the risk of stroke and ASCVD mortality, and are early markers of vascular impairment, such as intima-media thickness and coronary calcification. ${ }^{23}$

Both fluctuating BP and hypoxia have also an impact on the morpho-functional development of the myocardium; ${ }^{24}$ as a consequence, systemic hypertension, and in particular pre-eclampsia, may affect the myocardial development and its remodelling. In particular, offspring exposed to pre-eclampsia tend to show a geometrical pattern of the myocardium that goes toward a concentric remodelling, being 
characterized by an increased relative wall thickness (RWT), a reduced left ventricular (LV) end-diastolic diameter but a similar left ventricular mass index (LVMI) compared with offspring exposed to normal BP during pregnancy. ${ }^{25}$ If the mother is affected by gestational hypertension, the newborn is prone to have an augmented RWT without any changes in LV diameters. ${ }^{25}$ Furthermore, fluctuating systolic BP in the early stages of pregnancy, in particular between the eighth and the 18th weeks, is associated with larger LVMI and LV end-diastolic volume, and less optimal $\mathrm{LV}$ diastolic function, evaluated through the E/A ratio. ${ }^{25}$ Regarding the impact of pre-eclampsia on the ASCVD risk profile of the offspring, several studies have demonstrated that it might be associated with higher BP and body mass index (BMI) during childhood and adolescence, ${ }^{26}$ and an increased risk of stroke in adulthood, as compared with offspring not exposed to pre-eclampsia. ${ }^{27}$ These findings may need to be considered in future primary prevention strategies for cardiovascular disease. A recent meta-analysis confirmed the effects of pre-eclampsia on the BP profile of the offspring, although it has not been yet clarified whether its impact is more relevant for systolic, as the majority of the studies have attested, or diastolic BP. ${ }^{28}$

Therefore, the current evidence suggests that pregnancy-related hypertensive disorders might significantly influence the ASCVD risk profile of the offspring, supporting the concept that an appropriate management and treatment of these conditions may play a relevant role as a preventive measure.

\section{Maternal diabetes}

Changes during pregnancy do not affect only the cardiovascular system but also the hormonal and metabolic profile. Indeed, after the first trimester the glucose blood levels tend to progressively increase, sustained by a physiological insulin-resistance, which guarantees an optimal glucose supply to the foetus. However, when this insulin-resistance is exacerbated, these metabolic compensations go beyond the normal range, resulting in maternal hyperglycaemia and gestational diabetes (GDM). GDM is defined as glucose intolerance that develops in the second or third trimester of pregnancy and that leads to maternal hyperglycaemia. ${ }^{29}$ GDM determines an increased risk of pregnancy complications, such as shoulder dystocia, macrosomia and foetal hypoglycaemia, together with negative long-term effects for the mother and the child. ${ }^{30}$ In particular, diabetic mothers have a higher risk of developing type II diabetes mellitus and ASCVD later in life. ${ }^{31}$ Furthermore, the rate of obesity, systemic hypertension, metabolic syndrome and obesity increases and these conditions are known to have a great impact on cardiovascular health. ${ }^{32}$ As in gestational hypertensive disorders, the intra-uterine exposure to a diabetic environment could determine a cardiac remodelling in the exposed offspring. Indeed, GDM has been associated with an increased rate of cardiac abnormalities, ${ }^{33}$ with the most common anomaly being an asymmetrical myocardial hypertrophy, often localized in the interventricular septum. ${ }^{34}$ Diastolic dysfunction is the primarily manifestation of this foetal cardiovascular dysfunction, which later might evolve into systolic impairment. ${ }^{35}$ An early ventricular dysfunction caused by intra-uterine exposure to a diabetic environment has been confirmed also by echocardiographic studies analysing myocardial deformation: Miranda et al. have confirmed that during the third trimester diastolic strain rate values of both ventricles were significantly lower in exposed versus unexposed foetuses and systolic function of the right ventricle (RV) is more likely to be impaired compared with the LV, as demonstrated by a lower global longitudinal strain in the former. ${ }^{36}$ Several possible explanations have been proposed, such as the central role of the RV in the foetal circulation and the correlation between RV mass alterations and the need for postnatal ventilation.

Therefore, the current evidence suggests that intrauterine exposure to a diabetic environment may have an impact on biventricular geometry and function later in life, underlining the need for an adequate control of maternal glycaemia to reduce the negative effects for the foetus of hyperglycaemia.

\section{Low-grade systemic inflammation}

Over the years attention has been focused on the role of systemic inflammation as an important determinant of ASCVD. The relevance of this concept has been tested in several lipid-lowering trials, demonstrating the presence of a certain degree of residual systemic inflammation despite drug treatment, as suggested by high levels of high-sensitivity reactive C-protein, in patients with a prior myocardial infarction, which was also associated with a higher risk of re-infarction, stroke and mortality. ${ }^{37}$ A latent systemic inflammation can be found also during pregnancy if pathological changes occur and might have a relevant repercussion on the offspring. Indeed, pre-eclampsia is characterized by placental and systemic inflammation, which tends to persist even after delivery and which causes a certain level of endothelial dysfunction. ${ }^{38}$ Moreover, a study has shown that female offspring of mothers affected by type I diabetes mellitus are characterized by a higher level of the soluble form of the haptoglobin-haemoglobin scavenger receptor CD163, a marker of inflammation. ${ }^{39}$ Notably, recent investigations have associated 
this marker with obesity-related low-grade inflammation and future development of type II diabetes mellitus. ${ }^{40,41}$ Indeed, inflammation plays a significant role even later in life, since the presence of visceral fat, as it occurs with obesity, is a source of pro-inflammatory cytokines, ${ }^{42}$ which are able to induce endothelial impairment, paving the way for arterial hypertension to occur.

\section{Is it possible to prevent adverse cardiac remodelling?}

Hypertensive disorders during pregnancy and GDM have a great impact on the ASCVD risk profile of the exposed offspring and this prompts the question of whether these effects might be prevented or at least reversed by taking into account particular preventive measures. Currently, although the evidence is still scarce, the role of physical activity and nutrition predominates in preventing the onset of these disorders. Unfortunately, there is a lack of large scale clinical trials investigating the hypothesis that physical activity and/or diet might reverse the foetal cardiac remodelling once it has occurred. Therefore, beyond the general beneficial effects of an adequate nutrition and physical activity, the most important strategy to apply in this phase of the life is to prevent metabolic and hypertensive diseases during pregnancy. If prenatal exercise has been found to be a valuable measure in reducing the incidence of pre-eclampsia, ${ }^{43}$ its role in GDM is still controversial. Indeed, physical exercise combined with diet was shown to reduce the risk of developing GDM only if the mother was overweight or obese, ${ }^{44}$ whereas other authors have demonstrated that physical activity and diet are effective in reducing the incidence of macrosomia, but not plasma glucose levels. ${ }^{45}$ However, a recent meta-analysis, including 25 studies investigating the relation between exercising and GDM and/or abnormal glucose tolerance, attested that regular physical activity before pregnancy and in its early stages is effective in reducing the risk of GDM. ${ }^{46}$ These findings suggest the need for randomized clinical studies that investigate the role of these two preventive measures, also accounting for possible confounding factors. However, the evidence currently available supports physical activity and nutrition as effective strategies to mitigate the negative effects of pre-eclampsia and GDM.

\section{Pre-terms and cardiovascular outcome}

Considering the increasing level of perinatal care, the number of pre-term births has been raising over recent years, involving around 15 million babies each year across the world. ${ }^{47}$ Several causes that lead to an early delivery have been identified, and one of the most important is pre-eclampsia. However, aside from the possible implications of pre-eclampsia, as mentioned earlier, several studies have attested that preterms present a peculiar cardiac remodelling. Indeed, they tend to have an increased LV mass (LVM) with reduced volumes of both ventricles and a reduced apical displacement. ${ }^{39}$ Furthermore, the severity of these alterations might be correlated with the degree of prematurity. ${ }^{48}$ However, the cardiovascular phenotype of pre-term-born young adults is characterized by a preserved LV ejection fraction at rest, despite the geometrical alterations regarding the ventricles, even if a decrease in LV ejection fraction during increasing exercise has been demonstrated. ${ }^{49}$ Huckstep et al. demonstrated that the severity of prematurity and LV length were predictive of this impaired LV response to physical activity. ${ }^{49}$ In pre-terms the RV seems to be more affected than the $\mathrm{LV}:{ }^{50}$ indeed, a lower $\mathrm{RV}$ myocardial systolic strain and a lower systolic RV ejection fraction have been reported. ${ }^{50}$ This phenomenon could be explained at least in part by the fact that the birth induces a profound shift from a hyperplastic growth pattern to a hypertrophic one, and the RV switches from being the major determinant of the cardiac output during foetal life to supply a lower pressure circulation. Another possible explanation, as mentioned above, is the requirement of mechanical ventilation after birth, according to the fact that one of the major complications in pre-terms is represented by respiratory dysfunction needing ventilation support, ${ }^{51}$ which greatly affects the right side of the heart. Although the clinical consequences of this peculiar cardiac phenotype in pre-terms have still to be entirely understood, recently a possible association between the risk of developing heart failure in young adults and the degree of prematurity has been hypothesized, based on the evidence that being born pre-term is associated with an altered myocardial development. ${ }^{52}$ The pathophysiological basis is still unclear, but animal models have demonstrated a greater cardiomyocyte hypertrophy and a higher interstitial myocardial fibrosis together with an abnormal cardiomyocyte maturation. ${ }^{53}$ Another clinical implication is the higher risk of future systemic hypertension and stroke in preterms, ${ }^{54}$ as compared with full term newborns, which is independent from the cardiac alterations ${ }^{48}$ Increased arterial stiffness, reduced cutaneous capillary density and endothelial function found in pre-terms have been indicated as factors contributing to these negative clinical events. ${ }^{55}$ However, these pathophysiological substrates are not entirely understood. Indeed, several controversies exist regarding the relation between premature birth and increased aortic stiffness: while some authors reported an increased arterial stiffness in preterms, others found no differences in this parameter, 
despite a higher $\mathrm{BP}$ in children born prematurely. ${ }^{55}$ Therefore, future studies are needed to investigate the impact of negative cardiac remodelling observed in preterms in defining the future ASCVD risk profile of the individual.

\section{Pre-term: is it possible to intervene to improve myocardial development?}

Since postnatal myocardial alterations in pre-terms are still evident in adulthood, ${ }^{56}$ it would be important to assess whether it is possible to intervene on the cardiac development, avoiding the persistence of this adverse cardiac remodelling later in life. The most consistent evidence regards the role of breast milk, since it is responsible for improving both cardiac function and structure in pre-term-born young adults, compared with fed formula. ${ }^{57}$ Other experimental studies on animals have attested the possible role of pharmacological therapies started right after birth, such as angiotensin receptor blockers, that might have a positive effect on cardiac development. ${ }^{58}$ Taking into account that physical exercise, in particular high-intensity exercise, induces a cardiac remodelling caused by haemodynamic changes, it would be of particular interest to investigate whether it could influence myocardial maturation in pre-terms and whether it could exert a beneficial effect on the global cardiovascular profile, lowering their risk of developing hypertensive disorders and positively affecting structural and functional myocardial remodelling.

\section{Primordial prevention}

The substrate for the development of ASCVD starts during the early stages of life and it is influenced over time by several, potentially modifiable, risk factors and behaviours. Furthermore, all the efforts made towards ASCVD prevention might have a relevant impact later in life, further strengthening the role of primordial prevention. In 2010, the American Heart Association described ideal cardiovascular health by seven ASCVD health metrics, suitable both for children and adults (Table 1). ${ }^{59}$ These metrics included four behavioural criteria defined as healthy, such as non-smoking, having a BMI within normal range, being physically active and eating a healthy diet, and three health factors, including normal BP, cholesterol profile and plasma glucose level. ${ }^{59}$ It has been reported that increasing the number of these ideal metrics during childhood significantly reduces the incidence of arterial hypertension, metabolic syndrome and dyslipidaemia. ${ }^{60}$ Furthermore, the number of metrics achieved is inversely correlated with carotid intima-media thickness (IMT) and is positively associated with arterial
Table I. Cardiovascular health metrics described by the American Heart Association, suitable both for children and adults.

\begin{tabular}{ll}
\hline Behavioural criteria & Health factors \\
\hline Non-smoking & Normal blood pressure \\
BMI within range & Normal cholesterol profile \\
Being physically active & Normal plasma glucose level \\
Healthy diet & \\
\hline
\end{tabular}

For details see Lewandowski et al. ${ }^{50}$

BMI: body-mass index.

elasticity. ${ }^{61}$ This evidence highlights the great impact on arterial wall of several cardio-metabolic risk factors developed during childhood and adolescence. ${ }^{62}$ Notably, a relevant influence on a child's ASCVD risk profile is the genetic patrimony inherited from the parents. Indeed, possible mutations in genes that exert some effects on the individual's behaviour might have a relevant influence in defining the risk profile. Several studies have demonstrated the impact of genetics on cardiovascular risk and, although an analysis of the current literature on this topic is beyond the scopes of this review, we have at least to take into account that several studies have demonstrated that the correlation between a child's BMI and the BMI of the family members is due largely to genetic influences. ${ }^{63,64}$ Another recent study has reported that genetic susceptibility to develop overweight and obesity might be correlated with appetitive traits in children, which therefore might benefit from family counselling. ${ }^{65}$ This result stresses the tight relationship between the genetic component and the environment, with a close and reciprocal influence. For this reason, although the genetic patrimony cannot be directly influenced, it is important to stress the role of healthy behaviours in the family, in order to try to at least partially counteract inherited genetic traits that increase the risk of developing ASCVD risk factors.

A cornerstone for supporting primary prevention is represented by The Special Turku Coronary Risk Factor Intervention Project for Children (STRIP) Study, which has investigated the possible health repercussions during adulthood of lifestyle changes during the early stages of life. ${ }^{61}$ The authors demonstrated that dietary counselling in children has a beneficial effect on lowering plasma lipids in the long term not only in the child but also in the parents, and in particular in the mother, ${ }^{66}$ and it might improve insulin sensitivity during adolescence. ${ }^{67}$ Regarding the effects on $\mathrm{BP}$, as Gillman pointed out in a recent editorial, ${ }^{68}$ there are three major trials supporting primordial prevention: the STRIP study ${ }^{61}$ the Sodium Restriction Trial ${ }^{69}$ and the 
Promotion of Breastfeeding Intervention (PROBIT) trial. $^{70}$ The intervention period in the first trial started in infancy and continued through adolescence, whereas the other two trials were limited to infancy. Both the STRIP and the sodium restriction trial showed that counselling on $\operatorname{diet}^{61}$ and lower-sodium formula and baby foods ${ }^{69}$ respectively are associated with lower BP later in life. Conversely, breastfeeding did not show the same beneficial impact, as attested in the PROBIT study. ${ }^{70}$

Physical activity represents a main pillar of ASCVD prevention. Indeed, in children with levels of cholesterol above the normal range, physical activity influenced a decreasing trend of plasma lipids and lower BP, in a three-year period. ${ }^{71}$ Furthermore, similar results were obtained in obese children, since regular exercise induced a reduction of arterial stiffness, abdominal fat and BP, during three- and six-month follow-up. ${ }^{72}$ The positive effects on childhood vasculature ${ }^{73}$ were particularly important when long-term effects were evaluated, since arterial stiffness is one of the major determinants of high BP. Indeed, moderate intensity physical activity might prevent clustering of ASCVD risk factors, ${ }^{74}$ stressing the importance of starting exercising as soon as possible. Several studies have also demonstrated that exercise-induced cardiac remodelling can occur early in pre-adolescents and adolescents with a significant adaptation of the cardiovascular system starting in the early phases of the career of a young athlete. ${ }^{75,76}$

Over the years, beyond diet and physical exercise, a growing evidence on the efficacy of family-based interventions in ASCVD risk prevention has been demonstrated. ${ }^{77}$ Indeed, better results have been achieved in terms of preventing and reducing childhood obesity when both parents or all family members were involved in this approach, compared with strategies targeted only on the child. ${ }^{78}$ Parents have a highly influential role in supporting and encouraging children to adhere to a healthy lifestyle, not only in terms of nutrition and physical exercise, but also on the management of media use and hours of sleep. ${ }^{79}$ Furthermore, these strategies have a positive impact not only on the ASCVD risk profile of the child, but also in their parents, which makes these interventions particularly valuable and worth endorsing. ${ }^{80}$

Taken together, the current evidence strongly supports the role of diet, regular physical exercise and family-based intervention early in the life of a child to control ASCVD risks and to prevent adverse cardiac events later in life.

\section{Adolescents and young adults}

In both children and adolescents with multiple risk factors, an accelerated rate of atherosclerosis in adulthood, documented by an increased IMT, has been demonstrated, underlying the importance of preventing the development of ASCVD risk factors at early stages in order to prevent their negative impact on cardiovascular health. ${ }^{81}$ The fundamental role of nutrition and physical exercise is confirmed also at this stage of life. Indeed, the prospect of clustered metabolic risk was found to be higher in sedentary adolescents compared with adolescents practising leisure-time physical activity. ${ }^{82}$ Furthermore, Ekelund et al. showed that the time spent practising moderate-to-vigorous physical activity during childhood and adolescence was associated with a better ASCVD risk profile, independently on the amount of sedentary time. ${ }^{83}$ These beneficial effects can be extended also to endothelial function and IMT, since the progression of the latter is reduced in adolescents engaged in exercise practice. ${ }^{84}$ Considering that both endothelial function and IMT are the first steps towards an overt atherosclerosis, these results regarding the effects of exercise are particularly promising in the context of ASCVD prevention. Adolescents practising physical exercise have wider arteriolar diameter ${ }^{85}$ together with a greater arteriolar/venular ratio, ${ }^{86}$ both indicators of a better retinal circulation and therefore of the systemic microcirculation, as compared with sedentary adolescents. In agreement with these findings, a recent study has proved a positive effect on the retinal microcirculation promoting combined aerobic and coordinative exercise during the school day, suggesting that the latter might be a valuable measure to implement cardiovascular health. ${ }^{87}$ In 2018 Williamson et al. designed a randomized controlled trial, named Trial of Exercise to Prevent HypeRtension in young Adults, to investigate the effects of lifestyle changes, in particular physical activity, on the management of BP. The results of this trial are still non-available due to the expected 12-month follow-up, ${ }^{88}$ but they will help expand the evidence on this topic, increasing our knowledge and stimulating further researches and supportive clinical strategies.

Regarding nutrition, a well-balanced and healthy diet during adolescence has been associated with a lower risk of developing ASCVD risk factors. ${ }^{89}$ Although in the literature controversies exist regarding the possible benefits of fruit and vegetables, mainly due to the heterogeneity in assessing and classifying the consumption, a recent investigation highlighted that avoiding sweetened beverages and following a diet rich in fruit and vegetables might exert a cardioprotective effect in adolescents. ${ }^{90}$ Despite the complexity of this association, several studies have attested a relation between an unhealthy dietary pattern and a less favourable cardiac and metabolic profile in both children and adolescents. ${ }^{91}$ Accordingly, combining a healthy diet 
with regular physical exercise further reduces ASCVD risk in adolescents. ${ }^{92}$

Taken together, data currently available strongly support a healthy diet and physical exercise as relevant preventive strategies to protect the cardiovascular system and to positively influence the ASCVD risk profile in adulthood. Unfortunately, despite this evidence, the number of adolescents and young adults that are sedentary, do not follow an appropriate diet and have a non-healthy lifestyle is increasing, describing an unexpected and underestimated risk scenario, even in professional athletes. ${ }^{93}$ The scientific and clinical community is therefore called to increase the overall sensibility among the general population, all of the health professionals, the politicians and all of the people that could be potentially involved in this process, identifying also the best strategy to reverse the current trend and to improve cardiovascular health and to promote the culture of early and primordial prevention.

\section{Limitations and conclusions}

ASCVD prevention should start as soon as possible, even before birth, considering that it can help minimize the negative effects of risk factors on cardiovascular health, lowering the likelihood of developing detrimental consequences which increase the chance of ASCVD later in life. Nutrition and physical exercise are two cornerstones of ASCVD prevention in the early phases of our life and evidence is progressively growing, even if, to date, solid and consistent data are still lacking. However, the current promising indications could represent a valuable starting point to design new studies aiming to investigate the effects of primordial and early primary prevention. Table 2 summarizes the current evidence and gap in literature on early cardiovascular prevention.

\section{Practical clinical implications}

The current evidence suggests the importance of preventing ASCVD risk factors in children and adolescents, in particular overweight and obesity, focusing the resources on nutrition and physical activity. These strategies should involve the whole family, not only the child, in order to be more effective and more beneficial for all members of the family. Furthermore, talking directly about weight should be discouraged, and promoting in general a healthy lifestyle rather than generalist concepts on overweight/obesity is recommended. ${ }^{94,95}$ Indeed, even well-intended comments on weight might be perceived negatively especially by adolescents, and are paradoxically linked to overweight. ${ }^{96}$ Furthermore, family interventions should be supported by public policies and strategies that incentivize healthy diet and physical activities. For instance, building parks, recreational spaces and biking trails could be effective, as well as making available and more affordable healthy food, effectively discouraging the consumption of so-called junk food. ${ }^{3}$ Table 3 summarizes the recommendations for a healthy lifestyle based on healthy eating habits, physical activity, appropriate screen time and sleep habits. Unfortunately, the current situation regarding healthy eating and proper physical exercise among children and adolescents in Europe does not reflect these recommendations. Compliance with the current recommendations varies considerably according to gender and different countries: ${ }^{97}$ for girls it ranged from $2 \%$ in Cyprus to $14.7 \%$ in Sweden, while for boys it ranged from $9.5 \%$ in Italy to $34.1 \%$ in Belgium. ${ }^{97}$ In general, boys were found to be more active than girls, who were instead engaged more commonly in sedentary activities, and an inverse correlation between exercising and BMI was reported. ${ }^{97}$ Regarding adherence to a healthy diet, a higher than expected consumption of free sugars, mainly from fruit juices and soft drinks, has been found. ${ }^{98}$ Finally, a low adherence to the Mediterranean diet among children living in Mediterranean countries has been demonstrated ${ }^{99}$ and it has been recently attested that almost one in four young obese sudden death patients show some degree of coronary artery disease, ${ }^{100}$ further stressing that medical doctors, scientific communities and politicians should promote the most appropriate preventive measures to counteract this unfavourable trend that will have a negative impact on major adverse cardiovascular events in the coming years. In Table 3 general recommendations for a healthy lifestyle and the prevention of overweight and obesity in children, based on healthy eating habits, physical activity, appropriate screen time and sleep habits, are reported. ${ }^{101-103}$

\section{Future directions}

The clinical relevance of antenatal, perinatal and primordial prevention is still developing, and it has been growing over recent decades according to the latest evidences. However, data currently available are still few and more research is need also to identify the best practical approach to reach the aims imposed by cardiovascular prevention. While the evidence on the beneficial effects of exercise in children is growing, few data are available about the best exercise modality to suggest in children and pre-adolescents. Moreover, considering its social relevance, it would be interesting to design a study that could help better understand the role of family behaviour and collective strategies in reducing physical inactivity and obesity in children and in promoting cardiovascular prevention. Future researches 
Table 2. Current evidence and gap in literature on early cardiovascular prevention.

Current evidence and gap on early cardiovascular prevention

Evidence

- Prenatal exercise can reduce the incidence of pre-eclampsia and gestational diabetes

- Breast milk plays a positive role in cardiac remodelling in pre-terms

- Higher number of health metrics in childhood reduces the incidence of arterial hypertension, metabolic syndrome and dyslipidaemia

- Dietary counselling is beneficial in children in lowering plasma lipids both in the child and the parents

- Family-based interventions are effective for cardiovascular prevention

- Moderate-to-vigorous physical activity during childhood and adolescence has positive effects on endothelial function and intimamedia thickness

- Combining physical exercise and healthy diet for the prevention of cardiovascular disease is crucial

Evidence gaps

- Optimal timing to start prevention measures is not yet clearly known

- The effects of physical exercise on cardiac remodelling in pre-term children are not clearly defined

- The best exercise modality in children is not yet clearly established

- The effects of mother's systemic inflammation on the cardiovascular risk profile of the offspring are not completely understood

- The most effective family-based intervention needs not be determined

Evidence gaps are defined as lack of strong data currently available supporting a recommendation. Their identification is intended also as a stimulus for the development of further research in this area.

Table 3. General recommendations for a healthy lifestyle and the prevention of overweight and obesity in children, based on healthy eating habits, physical activity, appropriate screen time and sleep habits.

Food and eating habits

- Incorporate a healthy diet made up of foods with low caloric density (vegetables, fruits, whole grains, low-fat dairy products, lean meats, lean fish, and legumes).

- Limit foods with high caloric density (fat-rich meats, fried foods, baked goods, sweets, cheeses and oil-based sauces).

- Eat breakfast daily.

- Encourage family meals in which parents and children eat together while sitting at a table without distractions (no television or other electronics).

- Limit eating out at restaurants, particularly fast food restaurants.

Beverages

- Avoid sugar-sweetened beverages (i.e. iced teas, sports drinks, energy drinks and juice drinks).

- Limit consumption of $100 \%$ fruit juices to appropriate portion sizes.

- Children less than 12 months of age should not drink juice unless clinically indicated.

- Encourage children to drink low-fat milk and plain tap water.

- Children less than 12 months of age should not drink cow's milk.

- Children I-2 years of age should drink whole milk because fat is needed for healthy growth and development. However, in the context of obesity or strong family history of obesity, dyslipidaemia or other cardiovascular risk factors then reduced fat $(2 \%)$ milk is recommended.

- Children older than age two years should transition to low-fat (1\%) or fat-free milk.

Physical activity, screen time and sleep habit

- Encourage daily practice of physical activity (from moderate to vigorous).

- Limit non-educational screen time to less than $2 \mathrm{~h}$ per day.

- Remove televisions and other media from the bedroom.

- Foster healthy sleep patterns.

See Anderson, ${ }^{101}$ Heyman et al. ${ }^{102}$ and Holt et al. ${ }^{103}$ for details.

on the proper and optimal time to start prevention measures, their duration and maintenance in adulthood, and the most effective interventions according to the different ages will guide in the next years the best clinical practice and the political strategies to cope with cardiovascular disease.

\section{Author contribution}

FD, CS, MC, AC, MMC, SDF, AG, RL, PP, PP, GP, RP, PS, AZ, EI and AVM contributed to the conception or design of the work. FD, FA and CS drafted the manuscript. FD and SC contributed to the acquisition, analysis, or interpretation of data for the work. All the authors critically 
revised the manuscript. All gave final approval and agree to be accountable for all aspects of work ensuring integrity and accuracy.

\section{Declaration of conflicting interests}

The author(s) declared no potential conflicts of interest with respect to the research, authorship, and/or publication of this article.

\section{Funding}

The author(s) received no financial support for the research, authorship, and/or publication of this article.

\section{References}

1. GBD 2015 Mortality and Causes of Death Collaborators. Global, regional, and national life expectancy, all-cause mortality, and cause-specific mortality for 249 causes of death, 1980-2015: A systematic analysis for the global burden of disease study 2015. Lancet 2016; 388: 1459-1544.

2. Darnton-Hill I, Nishida C and James WP. A life course approach to diet, nutrition and the prevention of chronic diseases. Public Health Nutr 2004; 7: 101-121.

3. Weintraub WS, Daniels SR, Burke LE, et al. Value of primordial and primary prevention for cardiovascular disease: A policy statement from the American Heart Association. Circulation 2011; 124: 967-990.

4. Strasser T. Reflections on cardiovascular diseases. Interdiscip Sci Rev 1978; 3: 225-230.

5. Barker DJ, Winter PD, Osmond C, et al. Weight in infancy and death from ischaemic heart disease. Lancet 1989; 2: 577-580.

6. Barker DJ. The foetal and infant origins of inequalities in health in Britain. J Public Health Med 1991; 13: 64-68.

7. Durmus B, Kruithof CJ, Gillman MH, et al. Parental smoking during pregnancy, early growth, and risk of obesity in preschool children: The Generation R Study. Am J Clin Nutr 2011; 94: 164-171.

8. Durmus B, Ay L, Hokken-Koelega AC, et al. Maternal smoking during pregnancy and subcutaneous fat mass in early childhood. The Generation R Study. Eur $J$ Epidemiol 2011; 26: 295-304.

9. Dior UP, Lawrence GM, Sitlani C, et al. Parental smoking during pregnancy and offspring cardio-metabolic risk factors at ages 17 and 32. Atherosclerosis 2014; 235: 430-437.

10. Mamun AA, O'Callaghan MJ, Williams GM, et al. Maternal smoking during pregnancy predicts adult offspring cardiovascular risk factors - evidence from a community-based large birth cohort study. PLoS One 2012; 7: e41106.

11. Leybovitz-Haleluya N, Wainstock T, Landau D, et al. Maternal smoking during pregnancy and the risk of pediatric cardiovascular diseases of the offspring: A population-based cohort study with up to 18 -years of follow up. Reprod Toxicol 2018; 78: 69-74.

12. Zijlmans MAC, Beijers R, Riksen-Walraven MJ, et al. Maternal late pregnancy anxiety and stress is associated with children's health: A longitudinal study. Stress 2017; 20: 495-504.

13. Fan F, Zou Y, Tian H, et al. Effects of maternal anxiety and depression during pregnancy in Chinese women on children's heart rate and blood pressure response to stress. J Hum Hypertens 2016; 30: 171-176.

14. Jansen MAC, Dalmeijer GW, Saldi SR, et al. Pre-pregnancy parental BMI and offspring blood pressure in infancy. Eur J Prev Cardiol 2019; 26: 1581-1590.

15. Gaillard R, Bakker R, Willemsen SP, et al. Blood pressure tracking during pregnancy and the risk of gestational hypertensive disorders: The Generation R Study. Eur Heart J 2011; 32: 3088-3097.

16. Gallery ED. Pregnancy-associated hypertension: Interrelationships of volume and blood pressure changes. Clin Exp Hypertens B 1982; 1: 39-47.

17. Cnossen JS, Vollebregt KC, de Vrieze N, et al. Accuracy of mean arterial pressure and blood pressure measurements in predicting pre-eclampsia: Systematic review and meta-analysis. BMJ 2008; 336: 1117-1120.

18. Bakker R, Steegers EA, Hofman A, et al. Blood pressure in different gestational trimesters, fetal growth, and the risk of adverse birth outcomes: The Generation R Study. Am J Epidemiol 2011; 174: 797-806.

19. Jansen MA, Pluymen LP, Dalmeijer GW, et al. Hypertensive disorders of pregnancy and cardiometabolic outcomes in childhood: A systematic review. Eur J Prev Cardiol 2019; 26: 1718-1747.

20. Powe CE, Levine RJ and Karumanchi SA. Preeclampsia, a disease of the maternal endothelium: The role of antiangiogenic factors and implications for later cardiovascular disease. Circulation 2011; 123: 2856-2869.

21. Khan F, Belch JJ, MacLeod M, et al. Changes in endothelial function precede the clinical disease in women in whom preeclampsia develops. Hypertension 2005; 46: 1123-1128.

22. Li LJ, Cheung CY, Ikram MK, et al. Blood pressure and retinal microvascular characteristics during pregnancy: Growing Up in Singapore Towards Healthy Outcomes (GUSTO) Study. Hypertension 2012; 60: 223-230.

23. Kawasaki R, Cheung N, Wang JJ, et al. Retinal vessel diameters and risk of hypertension: The Multiethnic Study of Atherosclerosis. $J$ Hypertens 2009; 27: 2386-2393.

24. Davis EF, Newton L, Lewandowski AJ, et al. Preeclampsia and offspring cardiovascular health: Mechanistic insights from experimental studies. Clin Sci (Lond) 2012; 123: 53-72.

25. Timpka S, Macdonald-Wallis C, Hughes AD, et al. Hypertensive disorders of pregnancy and offspring cardiac structure and function in adolescence. $J$ Am Heart Assoc 2016; 5: e003906.

26. Davis EF, Lazdam M, Lewandowski AJ, et al. Cardiovascular risk factors in children and young adults born to preeclamptic pregnancies: A systematic review. Pediatrics 2012; 129: e1552-e1561.

27. Kajantie E, Eriksson JG, Osmond C, et al. Pre-eclampsia is associated with increased risk of stroke in the adult offspring: The Helsinki birth cohort study. Stroke 2009; 40: 1176-1180. 
28. Pinheiro TV, Brunetto S, Ramos JG, et al. Hypertensive disorders during pregnancy and health outcomes in the offspring: A systematic review. J Dev Orig Health Dis 2016; 7: 391-407.

29. American Diabetes Association. Classification and diagnosis of diabetes. Diabetes Care 2016; 39 Suppl 1: S13-S22.

30. Study Cooperative Research Group HAPO, Metzger BE, Lowe LP, et al. Hyperglycemia and adverse pregnancy outcomes. N Eng J Med 2008; 358: 1991-2002.

31. Bellamy L, Casas JP, Hingorani AD, et al. Type 2 diabetes mellitus after gestational diabetes: A systematic review and meta-analysis. Lancet 2009; 373: 1773-1779.

32. Carpenter MV. Gestational diabetes, pregnancy hypertension, and late vascular disease. Diabetes Care 2007; 30(Suppl. 2): S246-S250.

33. Lisowski LA, Verheijen PM, Copel JA, et al. Congenital heart disease in pregnancies complicated by maternal diabetes mellitus. An international clinical collaboration, literature review, and meta-analysis. Herz 2010; 35: 19-26.

34. Ullmo S, Vial Y, Di Bernardo S, et al. Pathologic ventricular hypertrophy in the offspring of diabetic mothers: A retrospective study. Eur Heart J 2007; 28: 1319-1325.

35. Donofrio MT, Moon-Grady AJ, Hornberger LK, et al. Diagnosis and treatment of fetal cardiac disease: A scientific statement from the American Heart Association. Circulation 2014; 129: 2183-2242.

36. Miranda JO, Cerqueira RJ, Ramalho C, et al. Fetal cardiac function in maternal diabetes: A conventional and speckle-tracking echocardiographic study. $J$ Am Soc Echocardiogr 2018; 31: 333-341.

37. Everett BM. Residual inflammatory risk: A common and important risk factor for recurrent cardiovascular events. J Am Coll Cardiol 2019; 73: 2410-2412.

38. Kvehaugen AS, Dechend R, Ramstad HB, et al. Endothelial function and circulating biomarkers are disturbed in women and children after preeclampsia. Hypertension 2011; 58: 63-69.

39. Boisen AB, Knorr S, Hansen TK, et al. Signs of lowgrade systemic inflammation in female offspring of women with type 1 diabetes: The EPICOM study. Diabetes Metab 2016; 42: 462-465.

40. Moller HJ, Frikke-Schmidt R, Moestrup SK, et al. Serum soluble CD163 predicts risk of type 2 diabetes in the general population. Clin Chem 2011; 57: 291-297.

41. Fjeldborg K, Christiansen T, Bennetzen M, et al. The macrophage-specific serum marker, soluble CD163, is increased in obesity and reduced after dietary-induced weight loss. Obesity (Silver Spring) 2013; 21: 2437-2443.

42. World Health Organization. Global status report on noncommunicable diseases. Geneva: World Health Organization, 2010.

43. Sorensen TK, Williams MA, Lee IM, et al. Recreational physical activity during pregnancy and risk of preeclampsia. Hypertension 2003; 41: 1273-1280.

44. Koivusalo SB, Rönö K, Klemetti MM, et al. Gestational diabetes mellitus can be prevented by lifestyle intervention: The Finnish gestational diabetes prevention study (RADIEL): A randomized controlled trial. Diabetes Care 2016; 39: 24-30.
45. Dodd JM, Turnbull D, McPhee AJ, et al. LIMIT Randomised Trial Group. Antenatal lifestyle advice for women who are overweight or obese: LIMIT randomised trial. BMJ 2014; 348: g1285.

46. Aune D, Sen A, Henriksen T, et al. Physical activity and the risk of gestational diabetes mellitus: A systematic review and dose-response metaanalysis of epidemiological studies. Eur J Epidemiol 2016; 31: 967-997.

47. World Health Organization. Preterm birth fact sheet, https://www.who.int/en/news-room/fact-sheets/detail/ preterm-birth (2018, accessed September 2019).

48. Lewandowski AJ, Augustine D, Lamata P, et al. Preterm heart in adult life: Cardiovascular magnetic resonance reveals distinct differences in left ventricular mass, geometry, and function. Circulation 2013; 127: 197-206.

49. Huckstep OJ, Williamson W, Telles F, et al. Physiological stress elicits impaired left ventricular function in pretermborn adults. J Am Coll Cardiol 2018; 71: 1347-1356.

50. Lewandowski AJ, Bradlow WM, Augustine D, et al. Right ventricular systolic dysfunction in young adults born preterm. Circulation 2013; 128: 713-720.

51. Brown MK and DiBlasi RM. Mechanical ventilation of the premature neonate. Respir Care 2011; 56: 1298-1311.

52. Carr H, Cnattingius S, Granath F, et al. Preterm birth and risk of heart failure up to early adulthood. J Am Coll Cardiol 2017; 69: 2634-2642.

53. Leeson $\mathrm{P}$ and Lewandowski AJ. A new risk factor for early heart failure: Preterm birth. $\mathrm{J}$ Am Coll Cardiol 2017; 69: 2643-2645.

54. Ueda $\mathrm{P}$, Cnattingius $\mathrm{S}$, Stephansson $\mathrm{O}$, et al. Cerebrovascular and ischemic heart disease in young adults born preterm: A population-based Swedish cohort study. Eur J Epidemiol 2014; 29: 253-260.

55. Bertagnolli M, Luu TM, Lewandowski AJ, et al. Preterm birth and hypertension: Is there a link? Curr Hypertens Rep 2016; 18: 28.

56. Lewandowski AJ, Augustine D, Lamata P, et al. Preterm heart in adult life: Cardiovascular magnetic resonance reveals distinct differences in left ventricular mass, geometry, and function. Circulation 2013; 127: 197-206.

57. Lewandowski AJ, Lamata P, Francis JM, et al. Breast milk consumption in preterm neonates and cardiac shape in adulthood. Pediatrics 2016; 138: e20160050.

58. Bertagnolli $\mathrm{M}$, Dios A, Béland-Bonenfant $\mathrm{S}$, et al. Activation of the cardiac renin-angiotensin system in high oxygen-exposed newborn rats: Angiotensin receptor blockade prevents the developmental programming of cardiac dysfunction. Hypertension 2016; 67: 774-782.

59. Bakker R, Steegers EA, Hofman A, et al. Blood pressure in different gestational trimesters, fetal growth, and the risk of adverse birth outcomes: The Generation R Study. Am J Epidemiol 2011; 174: 797-806.

60. Laitinen TT, Pahkala K, Magnussen CG, et al. Ideal cardiovascular health in childhood and cardiometabolic outcomes in adulthood: The Cardiovascular Risk in Young Finns Study. Circulation 2012; 125: 1971-1978.

61. Pahkala K, Hietalampi H, Laitinen TT, et al. Ideal cardiovascular health in adolescence: Effect of lifestyle intervention and association with vascular intima-media thickness and elasticity (the Special Turku Coronary 
Risk Factor Intervention Project for Children [STRIP] study). Circulation 2013; 127: 2088-2096.

62. Mikola H, Pahkala K, Niinikoski $H$, et al. Cardiometabolic determinants of carotid and aortic distensibility from childhood to early adulthood. Hypertension 2017; 70: 452-460.

63. Stunkard A, Harris J, Pedersen N, et al. The body-mass index of twins who have been reared apart. $N$ Engl J Med 1990; 322: 1483-1487.

64. Silventoinen K and Kaprio J. Genetics of tracking of body mass index from birth to late middle age: Evidence from twin and family studies. Obes Facts 2009; 2: 196-202.

65. De Lauzon-Guillain B, Koudou YA, Botton J, et al. EDEN Mother-Child Cohort Study Group. Association between genetic obesity susceptibility and mother-reported eating behaviour in children up to 5 years. Pediatr Obes 2019; 14: e12496.

66. Jaakkola JM, Pahkala K, Rönnemaa T, et al. Longitudinal child-oriented dietary intervention: Association with parental diet and cardio-metabolic risk factors. The Special Turku Coronary Risk Factor Intervention Project. Eur J Prev Cardiol 2017; 24: 1779-1787.

67. Oranta O, Pahkala K, Ruottinen S, et al. Infancy-onset dietary counseling of low-saturated-fat diet improves insulin sensitivity in healthy adolescents 15-20 years of age: The Special Turku Coronary Risk Factor Intervention Project (STRIP) study. Diabetes Care 2013; 36: 2952-2959.

68. Gillman MW. Primordial prevention of cardiovascular disease. Circulation 2015; 131: 599-601.

69. Hofman A, Hazebroek A and Valkenburg HA. A randomized trial of sodium intake and blood pressure in newborn infants. JAMA 1983; 250: 370-373.

70. Martin RM, Patel R, Kramer MS, et al. Effects of promoting longer-term and exclusive breastfeeding on cardiometabolic risk factors at age 11.5 years: A cluster-randomized, controlled trial. Circulation 2014; 129: 321-329.

71. Gidding SS, Barton BA, Dorgan JA, et al. Higher selfreported physical activity is associated with lower systolic blood pressure: The Dietary Intervention Study in Childhood (DISC). Pediatrics 2006; 118: 2388.

72. Farpour-Lambert NJ, Aggoun Y, Marchand LM, et al. Physical activity reduces systemic blood pressure and improves early markers of atherosclerosis in pre-pubertal obese children. J Am Coll Cardiol 2009; 54: 2396.

73. Idris NS, Evelein AM, Geerts CC, et al. Effect of physical activity on vascular characteristics in young children. Eur J Prev Cardiol 2015; 22: 656-664.

74. Andersen LB, Harro M, Sardinha LB, et al. Physical activity and clustered cardiovascular risk in children: A cross-sectional study (The European Youth Heart Study). Lancet 2006; 368: 299-304.

75. D'Ascenzi F, Pelliccia A, Valentini F, et al. Traininginduced right ventricular remodelling in pre-adolescent endurance athletes: The athlete's heart in children. Int $J$ Cardiol 2017; 236: 270-275.

76. D'Ascenzi F, Solari M, Anselmi F, et al. Atrial chamber remodelling in healthy pre-adolescent athletes engaged in endurance sports: A study with a longitudinal design. The CHILD study. Int J Cardiol 2016; 223: 325-330.

77. Foster C, Moore JB, Singletary CR, et al. Physical activity and family-based obesity treatment: A review of expert recommendations on physical activity in youth. Clin Obes 2018; 8: 68-79.

78. Golan M, Kaufman V and Shahar DR. Childhood obesity treatment: Targeting parents exclusively $v$. parents and children. Br J Nutr 2006; 95: 1008-1015.

79. Ash T, Agaronov A, Young T, et al. Family-based childhood obesity prevention interventions: A systematic review and quantitative content analysis. Int $J$ Behav Nutr Phys Act 2017; 14: 113.

80. Schwandt P and Haas GM. Family based prevention of cardiovascular disease risk factors in children by lifestyle change: The PEP Family Heart study. Adv Exp Med Biol 2019; 1121: 41-55.

81. Juonala M, Viikari JS, Kähönen M, et al. Life-time risk factors and progression of carotid atherosclerosis in young adults: The Cardiovascular Risk in Young Finns study. Eur Heart J 2010; 31: 1745.

82. Pahkala K, Heinonen OJ, Lagström H, et al. Clustered metabolic risk and leisure-time physical activity in adolescents: Effect of dose? Br J Sports Med 2012; 46: 131.

83. Ekelund U, Luan J, Sherar LB, et al. Moderate to vigorous physical activity and sedentary time and cardiometabolic risk factors in children and adolescents. JAMA 2012; 307: 704.

84. Pahkala K, Heinonen OJ, Simell O, et al. Association of physical activity with vascular endothelial function and intima-media thickness. Circulation 2011; 124: 1956.

85. Gopinath B, Baur LA, Wang JJ, et al. Influence of physical activity and screentime on the retinal microvasculature in young children. Arterioscler Thromb Vasc 2011; 31: 1233-1239.

86. Hanssen H, Siegrist M, Neidig M, et al. Retinal vessel diameter, obesity and metabolic risk factors in school children (JuvenTUM 3). Atherosclerosis 2012; 221: 242-248.

87. Ludyga S, Köchli S, Pühse U, et al. Effects of a schoolbased physical activity program on retinal microcirculation and cognitive function in adolescents. $J$ Sci Med Sport 2019; 22: 672-676.

88. Williamson W, Huckstep OJ, Frangou E, et al. Trial of exercise to prevent HypeRtension in young adults (TEPHRA) a randomized controlled trial: Study protocol. BMC Cardiovasc Disord 2018; 18: 208.

89. Dahm CC, Chomistek AK, Jakobsen MU, et al. Adolescent diet quality and cardiovascular disease risk factors and incident cardiovascular disease in middleaged women. J Am Heart Assoc 2016; 5: e003583.

90. Mellendick K, Shanahan L, Wideman L, et al. Diets rich in fruits and vegetables are associated with lower cardiovascular disease risk in adolescents. Nutrients 2018; 10: E136.

91. Rocha NP, Milagres LC, Longo GZ, et al. Association between dietary pattern and cardiometabolic risk in children and adolescents: A systematic review. $J$ Pediatr (Rio J) 2017; 93: 214-222.

92. Cuenca-García M, Ortega FB, Ruiz JR, et al. HELENA Study Group. Combined influence of healthy diet and 
active lifestyle on cardiovascular disease risk factors in adolescents. Scand J Med Sci Sports 2014; 24: 553-562.

93. D'Ascenzi F, Caselli S, Alvino F, et al. Cardiovascular risk profile in Olympic athletes: An unexpected and underestimated risk scenario. Br J Sports Med 2019; 53: 37-42.

94. Golden N, Schneider M and Wood C, Committee on Nutrition, Committee on Adolescence, Section on Obesity. Preventing obesity and eating disorders in adolescents. Pediatrics 2016; 138: e1.

95. Styne D, Arslanian S, Connor EL, et al. Pediatric obesity - assessment, treatment, and prevention: An endocrine society clinical practice guideline. J Clin Endocrinol Metab 2017; 102: 709-757.

96. Neumark-Sztainer DR, Wall MM, Haines JI, et al. Shared risk and protective factors for overweight and disordered eating in adolescents. Am J Prev Med 2007; 33: 359-369.

97. Konstabel K, Veidebaum T, Verbestel V, et al. IDEFICS consortium. Objectively measured physical activity in European children: The IDEFICS study. Int $J$ Obes (Lond) 2014; 38(Suppl. 2): S135-S143.

98. Graffe MIM, Pala V, De Henauw S, et al. Dietary sources of free sugars in the diet of European children:
The IDEFICS Study. Eur J Nutr, Epub ahead of print 4 April 2019. DOI: 10.1007/s00394-019-01957-y.

99. Pereira-da-Silva L, Rêgo C and Pietrobelli A. The diet of preschool children in the Mediterranean Countries of the European Union: A systematic review. Int J Environ Res Public Health 2016; 13: 572.

100. Finocchiaro G, Papadakis M, Dhutia H, et al. Obesity and sudden cardiac death in the young: Clinical and pathological insights from a large national registry. Eur J Prev Cardiol 2018; 25: 395-401.

101. Anderson KL. A review of the prevention and medical management of childhood obesity. Child Adolesc Psychiatr Clin N Am 2018; 27: 63-76.

102. Heyman $M$ and Abrams S, Section on Gastroenterology, Hepatology, and Nutrition, Committee on Nutrition. Fruit juice in infants, children, and adolescents: Current recommendations. Pediatrics 2017; 139: 1-8.

103. Holt K, Wooldridge N, Story M, et al. Nutrition supervision. Bright futures nutrition, 3rd ed. Elk Groove Village, IL: The American Academy of Pediatrics, 2011, pp.17-111. 